\title{
Routing Efficiency Evaluation in Wireless Sensor Networks
}

\author{
Mina Malekzadeh \\ Faculty of Electrical and \\ Computer Engineering, $\mathrm{Ha}$ \\ Sabzevari University \\ Sabzevar, Iran
}

\author{
Amirhamzeh Nazari \\ Engineering Faculty of \\ Golestan \\ University, Gorgan, Iran
}

\author{
M.H. Shahrokh Abadi \\ Faculty of Electrical and \\ Computer Engineering, Hakim \\ Sabzevari University \\ Sabzevar, Iran
}

\begin{abstract}
Today wireless sensor networks (WSNs) have gained grate popularity and are widely used in variety of areas due to their unique features. WSNs are deployed in an ad-hoc manner and consist of a large number of tiny sensor nodes. The sensor nodes communicate with each other through the routes established by the routing algorithms. Routing is considered as a challenging task in WSNs because it can highly affect the overall performance of these networks. This paper focuses on performance evaluation of two popular routing algorithms: Ad-Hoc on Demand Distance Vector (AODV) and Dynamic Source Routing (DSR). Simulations are performed using NS2 to evaluate and compare the efficiency of these routing algorithms under different network conditions.
\end{abstract}

\section{Keywords}

Scalability, Routing efficiency, Nodes density

\section{INTRODUCTION}

Routing is a process to establish and maintain routes which are used to move packets between sources and destinations more quickly and efficiently. Two common routing algorithms used in wireless sensor networks (WSNs) are Ad Hoc On-demand Distance Vector (AODV) and Dynamic Source Routing (DSR).

\section{AODV protocol}

When a source node $S$ needs a route to destination node $\mathrm{D}$, it broadcasts a ROUTE REQUEST message to all its neighbors. The message includes information such as source IP, destination IP, lifespan of the message, and destination Sequence Number (SN). The $\mathrm{SN}$ helps in identifying the freshness of the route to the destination so that higher sequence number for a node represents fresher information. When the ROUTE REQUEST reaches a node with a route to $\mathrm{D}$ with sequence number greater than or equal to it, then that node generates a ROUTE REPLY that contains the number of hops for D. Since there might be several nodes sending back the ROUTE REPLY, the needy node $\mathrm{s}$ uses the route that has the least number of hops to the destination and greater sequence number, over the other nodes. Thus, AODV determines the route from the source to the destination by independent hop-by-hop routing decisions made by each node. Any intermediate node always tries to keep a list of all its next hops.

\section{DSR protocol}

a complete ordered list of nodes information for the path from the source to the destination is included in the header of the request. A given intermediate node simply forwards data packets to the specified next node. The sender identifies the entire route node into which the packet has to pass through to reach the destination. Then the sender lists the route in the packet's header so that the next intermediate node can be identified by the address on the way to the destination host. Fresh routing information is not needed to be maintained in the intermediate nodes since all the routing decisions are contained in the packets by themselves. This results in less routing overhead for DSR than AODV.

This study is conducted to evaluate and compare the performance of DSR and AODV by using NS2 simulation tool. The effects that sensor nodes density and traffic patterns have on the efficiency of the protocols are taken into account. The remainder of this paper is structured as follows. Section 2 surveys related works. In Section 3 the simulation scheme and scenarios are presented. Section 4 presents and discusses the simulation results. Finally, Section 5 concludes the work.

\section{RELATED WORKS}

In this section we briefly mention the most significant articles related to our work. There has been extensive comparativebased research in routing protocols. In [1] the authors investigated three routing protocols as DSR, OLSR, and AODV for VoIP telephony application. The protocols are compared in terms of route discovery time, total packet dropped, and throughput for 50 nodes in network. The simulation is performed using OPNET simulator. Their work differs from us in different aspects including the simulation tool, node density, and the network load.

E.Chatzistavros and G.Stamatellos [2] compared the performance of three protocols as DBF, ZRP, and DSR. They examined the effect of the buffer size and nodes mobility on the performance of the protocols using Qualnet simulator. In compare to their work, we conduct our investigation based on different types of routing protocols using a different tool.

In [3] the authors compared four routing protocols as DSR, LAR, FSR, and AODV using GloMoSim simulator. On a network of 50 nodes the routing overhead, delay, and packet delivery ratio are used to evaluate the performance of the protocols. However, their work differs from us in terms of simulation tool, WSN environment, network load, and node density.

By Kumar et al, the performance of three routing protocols as DSR, CBRP, and AODV is evaluated [4]. Routing overhead, delay, and packet delivery ratio are used as the metrics for the evaluation. The simulations were performed using GloMoSim. By contrast, our study observes the protocols behavior by varying the nodes density and network load. Moreover, our study uses different simulation tool and evaluation metrics.

The authors [5] analyzed the performance of AODV and OLSR routing protocols by using OPNET simulator. The protocols are examined for traffic flow, data rates, and transmission power. However, this work evaluates different 
types of routing protocols based on different metrics and simulation tool.

A comparison over the performance of AODV, DSR, and OLSR protocols was provided in [6]. A simulation environment of 16 wireless nodes was developed. Average routing traffics sent and received, download response time, and delay were examined by using OPNET simulator. Their work differs from us in terms of the metrics, load density, node density, and the simulation tool. In addition to the above works, there are other studies $[7,8,9,10,13,14]$ each investigated different types of routing protocols, network parameters, and metrics.

Considering the related works, the main focus of this work is to develop a simulation-based framework to investigate the efficiency of the wireless sensor networks in presence of DSR and AODV routing protocols. We characterize the efficiency as better performance in terms of higher throughput and less delay. The key motivation of our work is to complement the existing studies with a simulation-based comparative assessment which can help selecting the right algorithm according to the networks requirements to achieve a better performance.

\section{SIMULATION MODEL}

We use NS2 to create a grid of $1000 \times 1000 \mathrm{~m}$ area as the WSN network environment. The scenarios and performance metrics to evaluate the routing protocols are described in the following.

\subsection{Simulation scenarios}

The scenarios are created concerning two key parameters including the density of the sensor nodes and traffic pattern in the simulation network. The goal is to take into account the effect of the node density and traffic pattern on the efficiency of the routing protocols in terms of the network end-to-end delay and throughput.

\section{Node density}

at the high level, the scalability characterizes the growth rate of the required network management resources with respect to the size of the network. The resources include the number of measurements needed to obtain an accurate estimate of the network performance and the time spent on computing the estimate [15]. Since, WSNs generally consist of a large number of wireless mobile sensor nodes, it is important to ensure the accurate management of the routing protocols as the WSN grows. In order to evaluate the scalability of the AODV and DSR protocols, we vary the sensor nodes density in the simulation environment so that the WSN size grows with $10,15,20,25,30,35$, and 40 sensor mobile nodes.

\section{Traffics pattern}

in the networks, the transmitted traffics have random patterns because different sensors nodes have different data to transmit. Different size of packets can directly affect the bandwidth consumed which results in changes in the maximum throughput achieved by WSNs. Therefore, to examine the impact of the size of traffics on the performance of WSN, variety sizes are transmitted as 100B, 200B, 300B, 400B, 500B, 600B, 700B, 800B, 900B, and 1000B

Using these two variable attributes, we compare the performance of DSR and AODV in the WSN. Table1 and table 2 in the following summarize the variable and constant parameters applied in our WSN simulation environment respectively.
Table1. Variable parameters used in the simulation

\begin{tabular}{ll}
\hline Parameter & Value \\
\hline Number of nodes & $10.15,20,25,30,35,40$ \\
Routing protocol studied & AODV and DSR \\
Packets size & 100B, 200B, 300B, 400B, \\
& $500 \mathrm{~B}, 600 \mathrm{~B}, 700 \mathrm{~B}, 800 \mathrm{~B}$, \\
& $900 \mathrm{~B}, 1000 \mathrm{~B}$ \\
\hline
\end{tabular}

Table2. Constant parameters used in the simulation

\begin{tabular}{ll}
\hline Parameter & Value \\
\hline Channel type & WirelessChannel \\
Radio propagation & TwoRayGround \\
network interface type & WirelessPhy \\
MAC protocol & Mac/802_11 \\
interface queue type & Queue/DropTail/PriQueue \\
Mobility model & Random WayPoint \\
antenna mode & OmniAntenna \\
max packet in queue & 50 \\
x coordinate of topology & 1000 \\
y coordinate of topology & 1000 \\
Simulation time & $100 \mathrm{~s}$ \\
Traffic type & Constant Bit Rate (CBR) \\
Packet interval & $0.05 \mathrm{~s}$ \\
Parameter & Value \\
\hline
\end{tabular}

Simulation environment of one of the scenarios is presented in Fig.1.

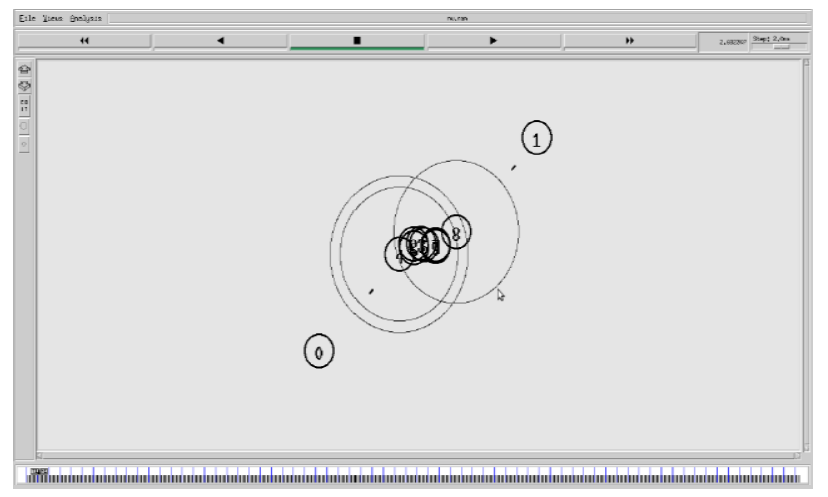

Fig1: Simulation environment

\subsection{Performance metrics}

In order to measure the impact of the above parameters on WSN, we investigate two metrics described as follows.

Throughput (T): is computed by dividing the amount of data received at the destination node by the time taken to arrive at this node:

$\mathrm{T}=\sum($ Received Packets * Packet size * 8)/ time taken

End-to-End delay (D): is considered as the time taken by a packet to travel from the sender node $\left(\mathrm{T}_{\mathrm{s}}\right)$ until it is 
successfully received at the receiver node $\left(\mathrm{T}_{\mathrm{r}}\right)$ by considering the propagation delay $\left(T_{p}\right)$ :

$\mathrm{D}=\mathrm{T}_{\mathrm{r}}-\mathrm{T}_{\mathrm{s}}+\mathrm{T}_{\mathrm{p}}$, where $\mathrm{T}_{\mathrm{p}}=$ distance between the source and destination / speed of light.

By combining the variable parameters and performance metrics, four distinct scenarios are examined as the states of the WSN.

\section{SIMULATION RESULTS AND DISCUSSION}

In this section, the simulation results of the four scenarios are presented.

\subsection{Scenario1: throughput while varying the nodes density}

In this experiment, 1000 bytes CBR packets with $0.05 \mathrm{~s}$ interval between the packets are transmitted between the users. The key motivation is to examine the impact of the node density, with the characteristics listed in Table2, on operation of the WSN in term of the maximum achieved throughput. The simulation results of this experiment are presented in Fig2.

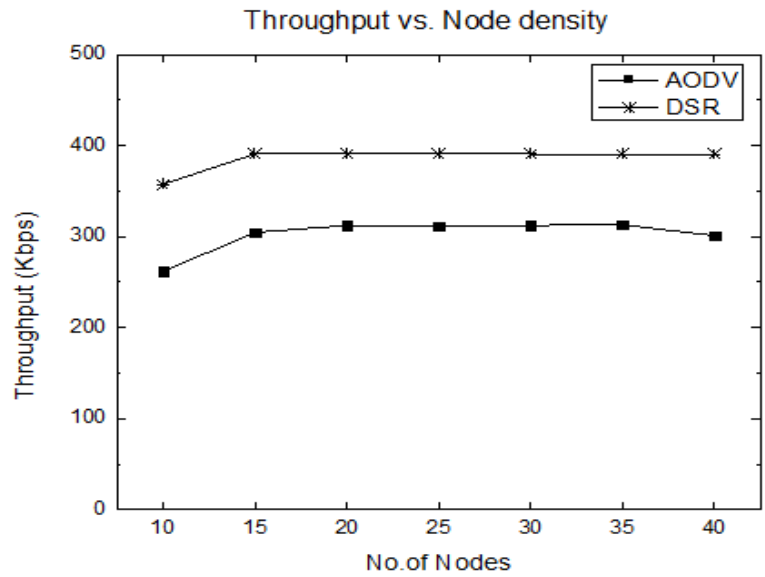

Fig2: WSN throughput with different nodes density

It is straightforward that the more nodes we have, the more hops will be contained in the route established between the sources and the destination. Based on the above results we can see that both protocols preserve the WSN scalability. They can accurately manage the network growing so that as the number of nodes increases the throughput stays in a relatively uniform state.

The above results also imply that regardless of the number of nodes, DSR is more efficient than AODV in term of the higher throughput. For 40 nodes, AODV shows less throughput than before which is related to the lost packets due to congestion. This shows DSR can handle network congestion more efficient than AODV.

\subsection{Scenario2: throughput while varying the packets size}

To demonstrate how the size of the packets routed by either AODV or DSR affect the throughput of WSN, we plot in Fig.3 the throughput as a function of the packets size ranging from 100 to 1000 bytes.

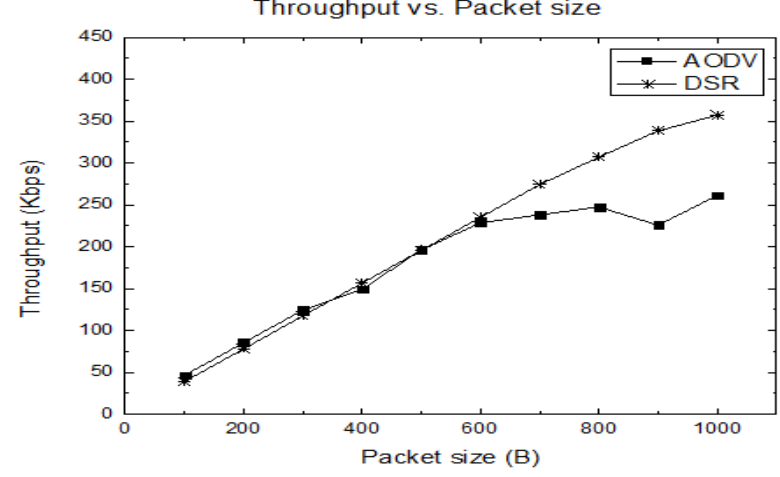

Fig3: WSN throughput with different packet sizes

The above results imply similar efficiency achieved by both AODV and DSR for packets smaller than 600B. For the small packets, the amount of throughput for both AODV and DSR increases quickly as the size of packets increases from 100 to 600 bytes. However, the results show that when the size of packets grows bigger, DSR is more efficient than AODV in term of higher throughput. Our results suggest that for the packets larger than 700B we still observe growing the throughput by DSR unlike AODV. The apparent slight decrease observed in the case of AODV is not statistically significant. So we can conclude that larger packets can degrade the efficiency of WSN when using AODV protocol.

\subsection{Scenario3: delay while varying the node density}

In this experiment, the nodes density is varied in the range between 10 to 40 nodes which are randomly placed over an area of $1000 \mathrm{~m} \times 1000 \mathrm{~m}$ WSN area. The experiment focuses on quantifying the impact of growing the WSN on efficiency of the AODV and DSR in term of delay. The results are presented in Fig.4.

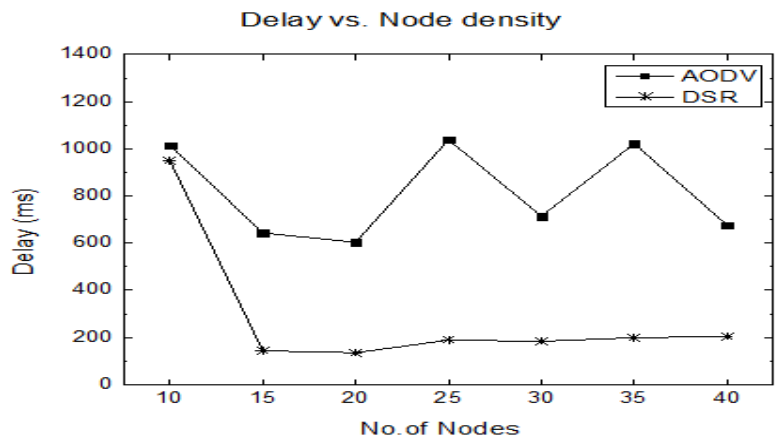

Fig.4: WSN delay with different nodes density

Based on the results, DSR performs almost the same while the number of nodes increases. However, the above graph shows that as the number of nodes increases, the AODV incurs higher delay to the WSN than DSR. The reason of higher delay for AODV is the frequent discovery process which is an inherent characteristic of this protocol.

\subsection{Scenario4: delay while varying the packets size}

In this experiment we send different packet sizes into the WSN to see the possible effects on the performance of the WSN. Fig.5 depicts how the delay is affected by the variations in the packet sizes for AODV and DSR. 


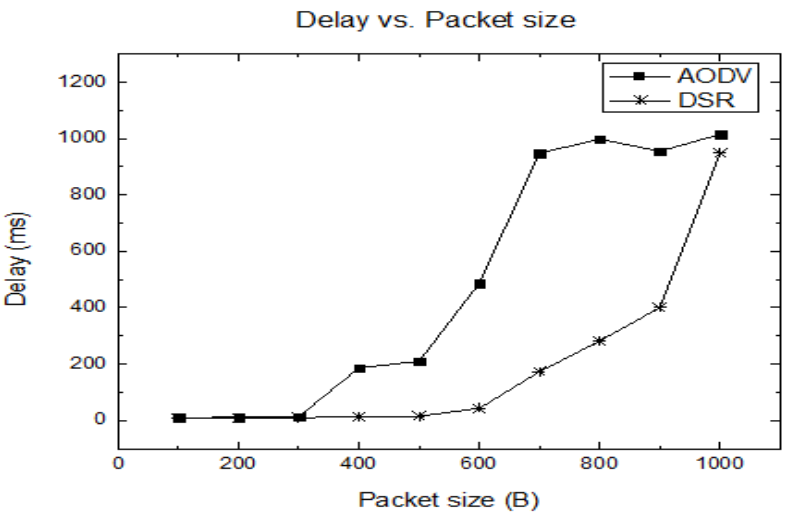

Fig.5: WSN delay with different packet sizes

From the simulation results it is observed that regardless of the type of routing protocol, a better performance in term of less delay is achieved by the WSN when the smaller packets are exchanged. The smaller packets, $100 \mathrm{~B}$ to $300 \mathrm{~B}$, provide the same amount of delay for DSR and AODV. However, as the size of packets grows, the delay caused by AODV grows higher than DSR. Additionally, we can find that when the size is between $300 \mathrm{~B}$ to $600 \mathrm{~B}$, the delay increases gradually. But after that, the delay increases more quickly. The reason is that the bigger packets need more time to process. This increases the amount of time they have to wait in the queue. Moreover, since larger packets induce congestion to the network, the packets will experience greater delay.

\section{CONCLUSION}

In this paper we have made a comparison between AODV and DSR routing protocols to evaluate their efficiency under different WSN conditions. The nodes density and traffic types have varied to measure their effects on the performance of the routing protocols in term of delay and throughput. The frequent discovery process as an inherent characteristic of the AODV protocol directly affects it performance. From the results it is concluded that DSR is more efficient in terms of higher throughput and less delay when it comes to scalability issue by changes in nodes density and load.

\section{REFERENCES}

[1] P.Singh, A.K. Sharma, and T. S. Kamal. Performance analysis of a routed ad hoc networks using different routing algorithms. International Journal of Computer Networking Wireless and Mobile Communications (IJCNWMC), Vol. 3, No. 1, pp. 117-122, 2013.

[2] E.Chatzistavros and G.Stamatellos. Comparative Performance Evaluation of Routing Algorithms in IEEE 802.11 Ad Hoc Networks. International Journal of Computer Science (IJCSI), Vol. 7, No. 4, pp. 1-10, 2010.
[3] N.Qadri and A.Liotta. A comparative analysis of routing protocols for manets. International Conference Wireless Applications and Computing (IADIS), pp.149-154, 2008.

[4] M.Kumar, R.Rishi, and D.K. Madan. Comparative Analysis of CBRP, DSR, AODV Routing Protocol in MANET. International Journal on Computer Science and Engineering (IJCSE), Vol. 02, No. 09, pp. 2853-2858, 2010.

[5] P.K.Bhardwaj, S.Sharma, and V.Dubey. Comparative Analysis of Reactive and Proactive Protocol of Mobile Ad-Hoc Network. International Journal on Computer Science and Engineering (IJCSE), Vol. 4, No. 07, pp.1281-1288, 2012.

[6] S.Lally and L.Trajković. Performance Analysis of Routing Protocols for Wireless Ad-HocNetworks. Simon Fraser University, Canada, pp. 1-8, 2011.

[7] D.Dembla and Y.Chaba. Performance analysis of on demand routing protocols of mobile ad hoc networks - A comparative study. The Journal of Computer Science and Information Technology, Vol. 3, No.1, pp. 30-34, 2006.

[8] P. Vijayalakshmi, V.Saravanan, and P. Ranjit Jeba. Mobile Ad Hoc Routing Protocols - A Comparative Performance Analysis by Diversifying the Nodes. International Journal of Computer Applications, Vol. 21, No. 5, pp. 42 - 47, 2011

[9] K.Karthikeyan, S.Appalabatla, M.Nirmala, and T.Tesfazghi. Comparative Analysis of Non-Uniform Unicast Routing Protocols for Mobile Adhoc Networks. International Journal of Advanced Research in Computer Science and Software Engineering, Vol. 2, No. 12, pp. 227-232, 2012.

[10] S.Kant and K.Kumar. Performance Analysis Of Dynamic Source Routing Protocol In Wireless Mobile Ad Hoc Network. International Journal of Engineering Research \& Technology (IJERT), Vol. 1, No. 10, pp. 1-7, 2012.

[11] F.Yu. A Survey of Wireless Sensor Network Simulation Tool.http://www1.cse.wustl.edu/ jain/cse56711/ftp/sensor/index.html

[12] M.Korkalainen, M.Sallinen, N.Kärkkäinen, and P.Tukeva. Survey of Wireless Sensor Networks Simulation Tools for Demanding Applications. IEEE International Conference on Networking and Services (ICNC), pp. 102-106, 2009.

[13] Chuanyi Ji. Measurement-Based Network Monitoring and Inference: Scalability and Missing Information IEEE journal on selected areas in communications, Vol. 20, No. 4, pp.714-725, 2002. 